\title{
NEW HIGH PERFORMANCE SCINTILLATORS BASED ON RE-ACTIVATED INSULATOR MATERIALS
}

\author{
A.J. Wojtowicz \\ Institute of Physics, N. Copernicus University, Grudziądzka 5, 87-100 Toruń, Poland \\ Chem. Dept., Boston University, 590 Commonwealth Ave., Boston, MA 02215, USA
}

In this paper we address the problem of the host-to-ion energy transfer in some RE-activated wide band gap materials excited by ionizing radiation. We argue that, despite the expected self-localization of holes, the dominant mechanism in efficient materials involves sequential trapping of both charge carriers (holes and electrons) by an activating RE-ion followed by a radiative recombination via the ion producing scintillation light. Selected experimental results are presented to illustrate how various energy transfer processes manifest themselves in the spectroscopy of scintillator materials. Experimental results combined with simple considerations are used to identify these RE-ions which are likely to act as hole or electron traps in tri- and difluorides, thus initiating the recombination sequence leading to efficient scintillation.

PACS numbers: $78.90 .+\mathrm{t}, 78.55 .-\mathrm{m}, 61.80 . \mathrm{Ed}, 29.40 .-\mathrm{n}$

\section{Introduction}

It is generally accepted that good performance of scintillators depends on efficiencies of three major steps: energy conversion, energy transfer, and luminescence $[1,2]$. The choice of the $\mathrm{Ce}^{3+} d-f$ transition usually warrants speed and efficiency of the luminescence step. Consequently, there are only two more requirements the material needs to fulfill in order to qualify as a good scintillator: it should efficiently transform an incoming high energy particle into a shower of lattice electronic excitations (electron-hole pairs and/or excitons), and energy transfer from these excitations to activating ions should be fast and efficient. Of these two steps transfer is more critical as demonstrated in the case of orthophosphates. Some of them, despite poor conversion, are still characterized by decent light outputs on the order of $15000-17000$ photons $/ \mathrm{MeV}$ [3].

Last years have witnessed an extensive search for new scintillator materials driven by large scale applications in high energy physics, nuclear medicine, security systems, and industry [4]. Better hosts for the $\mathrm{Ce}^{3+}$ ion as well as new activating 
ions providing even faster emissions in the VUV have been sought and tested. All these activities have contributed to the perception that in the long run no progress is possible without better understanding of scintillation mechanism and, in particular, of energy transfer mechanisms. The host-to-ion energy transfer in wide gap materials activated by RE-ions is the main subject of this paper.

\section{Host-to-ion encrgy transfer in wide band gap materials}

It has been long known that in photoconductive materials such as $\mathrm{ZnS}: \mathrm{Ag}$ the mechanism of the host-to-ion energy transfer is based on hole and electron transport and binding by the ion [5]. The same mechanism has been recently proposed in cerium-activated fluoride, orthophosphate, orthoaluminate, and oxide scintillator materials [6-8]. Nevertheless another mechanism has been also suggested. It is well known that in wide band gap materials self-trapping of holes followed by formation and decay of self-trapped excitons provides an important recombination mechanism [9]. It has been argued that the mechanism of energy transfer in many of cerium-doped oxide materials must, therefore, be based on nonradiative energy transfer from self-trapped and/or defect trapped excitons to $\mathrm{Ce}$ ions [10]. The rate of such a transfer should strongly depend on overlaps between excitonic emission and Ce-absorption and, indeed, some correlation between these overlaps and observed light outputs in different materials has been claimed and cited in support of this model [10]. Also in alkaline earth fluorides some authors reject any contribution from the host to the scintillation [11], while others neglect contribution from the charge carrier mediated energy transfer in favor of the excitonic mechanism [12]. In this paper we present direct spectroscopic evidence that, despite self-trapping, carrier transport and binding at the RE site is the dominant mechanism of the host-to-in energy transfer mechanism in $\mathrm{LuAlO}_{3}: \mathrm{Ce}$ and $\mathrm{CaF}_{2}: \mathrm{Eu}$. We also argue that the mechanism is likely to dominate in many other wide gap scintillator materials activated by RE-ions.

There are no doubt some scintillator materials which, when activated, are not likely to support any but the excitonic mechanism. In $\mathrm{CaWO}_{4}$ after ionization all initial core holes relax to almost completely immobile localized valence states of the $\mathrm{WO}_{4}^{2-}$ molecular ions. The resultant positively charged $\mathrm{WO}_{4}^{1-}$ species capture mobile electrons forming self-trapped excitons (STE), decay of which produces scintillation under ionizing excitation. Any competition for holes in order to destructively interfere with the scintillation process requires some transport of holes (relaxed or unrelaxed). Low hole mobilities serve therefore to the advantage by reducing a role of inadvertent hole traps. Electron traps, although not likely to introduce any competing radiative or nonradiative deexcitation channels, may play a role by delaying transport of electrons to ionized molecular groups. By the same token, however, any efficient radiative recombination process via some intentionally introduced activating ions is not likely and the only option left might be the nonradiative energy transfer [13].

IIowever, because of possible delay in self-trapping [14], "hot" holes may be able to move rapidly over large distances before they self-trap [15]. Even self-trapped holes, in many materials, are able to move reasonably fast by the hopping motion [16]. Sequential charge carrier binding of both unrelaxed and re- 
laxed (self-trapped) holes has been established in a well-known scintillator material NaI: Tl $[17,18]$. Despite the extremely short free hole lifetime on the order of 1-10 ps pointing to very efficient self-trapping [19], Abdrakhmanov et al. estimate that in $\mathrm{NaI}$ doped with $2 \times 10^{18} \mathrm{~cm}^{-3} \mathrm{Tl}$, at $300 \mathrm{~K}$, roughly comparable fractions of the total light yield come from three processes: consecutive trapping of unrelaxed holes and then of electrons by $\mathrm{Tl}^{1+}$ ions (prompt component), recombination of $\mathrm{Tl}^{0}$ with self-trapped holes (a rise time of only $300 \mathrm{ps}$ ), and recombination of electrons detrapped from the $\mathrm{Tl}^{0}$ traps, with $\mathrm{Tl}^{2+}$ (a rise time of about $4 \mathrm{~ns}$ ) [18]. Despite efficient self-trapping of holes scintillation in $\mathrm{NaI}: \mathrm{Tl}$ is based on radiative recombination of charge carriers via $\mathrm{Tl}$ ions, although relaxation processes and self-trapped hole mobilities are important and must not be neglected.

\section{Rare earth ions as radiative recombination centers}

As recently pointed out by Wojtowicz et al. $[7,8]$ (see also [20]), the condition that the RE-ion must fulfill in order to bind holes (or electrons) is to introduce an occupied (or empty) level above the valence band (below the conduction band). It was also observed that, although deep traps do provide large energy gains upon trapping of carriers, the capture process may nevertheless be severely impeded by the energy barrier $\Delta E_{\mathrm{b}}$ due to the electron-lattice coupling term in the total IIamiltonian of the system [7]. In this work, a simple one-configuration-coordinate model was introduced and described in detail. In the case of the hole trap

$$
\Delta E_{\mathrm{b}}=\Delta E^{2} / 4 \Delta E_{x}-\Delta E+\Delta E_{x}
$$

where $\Delta E$ is the optical trap depth (the energy of an optical transition $\mathrm{Ln}^{4+}+h \nu \rightarrow$ $\mathrm{Ln}^{3+}+h_{\mathrm{v}}$ which releases a valence hole $h_{\mathrm{v}}$ from the hole trap) and $\Delta E_{x}$, is the relaxation energy which increases the optical trap depth $\Delta E$ relative to the thermal equilibrium trap depth determining occupancy of the trap under steady state conditions. From Eq. (1), assuming that the relaxation energy $\Delta E_{x}$ is $1-1.5 \mathrm{eV}$, we estimate that the no-barrier thermal equilibrium trap depth is about 1-1.5 eV and the acceptable, low barrier, range of depths would be $0.5-3 \mathrm{eV}$. It is interesting to note that some RE impurities introducing band gap levels in the 1-5 eV gap materials may therefore be able to capture both electrons and holes in sequence with no light emission, acting as so-called "killer" (nonradiative recombination) centers. This is why such ions should be scrupulously avoided in any light emitting devices.

Although the approach summarized above is very simple we were able to demonstrate that it explains the good scintillator performance of Ce-activated orthophosphates and orthoaluminates. We found that nonradiative recombination involving $\mathrm{Ce}$ ions is highly unlikely and that Ce provides instead a good radiative recombination center for electron-hole pairs in those materials $[7,8]$. The filled $\mathrm{Ce}^{3+}$ level in these materials is located $2-2.5 \mathrm{eV}$ above the valence band so that the $\mathrm{Ce}^{3+}$ ions good hole traps and the recombination cycle is initiated by hole trapping. After a hole has been captured creating a $\mathrm{Ce}^{4+}$ ion, the position of the empty $\mathrm{Ce}^{3+}$ ground state level in 8-8.5 eV $E_{\mathrm{g}}$ materials must be at least $6-6.5 \mathrm{eV}$ below the conduction band $[7,8]$. Consequently the energy of a bound exciton formed upon trapping of an electron (at about $5.5-6.0 \mathrm{eV}$ ) is so high that nonradiative 
recombination is not likely to occur. Since there are five $f-d$ transitions extending over a broad spectral range it is very likely that the bound exciton transition will be covered facilitating an efficient energy transfer and $d-f$ emission. In the absence of overlapping $f-d$ transitions the bound excitonic emission may result as in the case of $Y b$-activated orthophosphates [6]. The available recombination energy for the sequence in which a hole is trapped first, and in larger band gap materials (fluorides), might be adequate to excite not only Ce but also VUV $d-f$ transitions of some other RE-ions. Since in trifluorides all of RE-ions except of Eu, $\mathrm{Gd}, \mathrm{Yb}$ and $\mathrm{Lu}$ have their 3+ level above the top of the valence band [21] they might, therefore, act as hole traps and radiative recombination centers. The idea of employing $d-f$ transitions of those ions in new scintillator materials certainly deserves more attention.

It is interesting to inquire about the possibility of the reversed sequence recombination via the RE-ion that is initiated by an electron rather than a hole capture. This type of recombination may be important in the case of RE-activated difluorides since all of the RE-ions have their $3+$ levels below the top of the valence band and none of them is expected, therefore, to initiate the recombination by capturing a hole. On the other hand, as shown by photoconductivity studies [22], most of RE-ions do have an empty 2+ charge state level below the conduction band introducing an electron trap capable of binding an electron and then attracting a mobile self-trapped hole. That such a process in $\mathrm{CaF}_{2}$ is possible has been demonstrated by thermoluminescence studies of Merz and Pershan [23]. Nevertheless, there is no report which would suggest that such a mechanism is active in any scintillator material.

\section{Excitons and charge carriers in $\mathrm{LuAlO}_{3}: \mathrm{Ce}$ and $\mathrm{CaF}_{2}: \mathbf{E u}$}

Preliminary results of luminescence studies on Ce-doped and undoped $\mathrm{LuAlO}_{3}$ under synchrotron VUV excitation have been briefly reported in Ref. [8]. More detailed account of this and subsequent recent work has also been given by Wiśniewski et al. [24], therefore only selected results are presented here.

In Fig. 1 we show emission and excitation spectra of undoped and Ce-doped $\mathrm{LuAlO}_{3}$. In emission, under $161 \mathrm{~nm}$ excitation, the undoped sample (Fig. 1a) shows a single broad band (designated STE) peaking at about $280 \mathrm{~nm}$. The spectra of the Ce-doped sample (Fig $1 \mathrm{~b}$ and c) are more complex showing the STE band with indents clearly caused by Ce-absorption and a well-known doublet band at 350 and $375 \mathrm{~nm}$ due to the $\mathrm{Ce}^{3+}$. The ratio of STE and Ce emissions changes strongly with the excitation wavelength. The STE excitation spectra (Fig. 1a and b) show distinct differences depending on whether $\mathrm{Ce}$ is present. In the undoped sample the spectrum consists of a single band peaking at about $152 \mathrm{~nm}$ with some contribution at $160 \mathrm{~nm}$, and with a tail extending toward shorter wavelengths on which some structure at 143 and $125 \mathrm{~mm}$ is imposed. Surprisingly, the same emission in the Ce-doped sample shows a different excitation spectrum consisting of a dominant band peaking at $160 \mathrm{~nm}$ and weaker contributions at 153 and $142 \mathrm{~nm}$. In the excitation spectrum of the Ce-emission itself (Fig. 1c) there is a prominent feature at $153 \mathrm{~nm}$, interpreted in Refs. [7] and [25] as a band-to-band transition.

The sharp excitation band of the $280 \mathrm{~nm}$ emission and its unusually large 


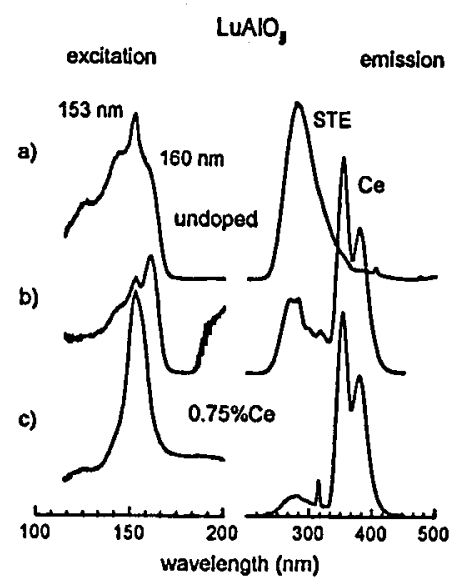

Fig. 1. The emission and excitation spectra of undoped and Ce-doped $\mathrm{LuAlO}_{3}$. The emission spectra were taken at $13 \mathrm{~K}$ (to show a resolved Ce-doublet) while excitation spectra were taken at room temperature to show thermally activated contributions to STE emission. For more detailed discussion see Ref. [24]. (a) Undoped $\mathrm{LuAlO}_{3}$; the emission spectrum under $161 \mathrm{~mm}$ excitation, the excitation spectrum was taken at $280 \mathrm{~nm}$. (b) Ce-doped (nom. 0.75\%) $\mathrm{LuAlO}_{3}$; the emission spectrum under $161 \mathrm{~nm}$, the excitation spectrum was taken at $275 \mathrm{~nm}$. (c) Ce-doped (nom. 0.75\%) $\mathrm{LuAlO}_{3}$; the emission spectrum under $153 \mathrm{~nm}$ excitation, the excitation spectrum was taken at $375 \mathrm{~nm}$.

Stokes shift $(3.7 \mathrm{eV})$ clearly suggest an excitonic origin. The emission at $280 \mathrm{~nm}$ must, therefore, be due to self- or defect-trapped excitons (as in $\mathrm{YAlO}_{3},[26]$ ); the peak at $160 \mathrm{~nm}$ corresponds to creation of free (band) excitons, while the shorter wavelength tail is due to free electron-hole pairs. The difference in the STE excitation spectra in undoped and Ce-doped samples, showing a strong reduction of free electron-hole pair contribution to STE in the Ce-doped sample, suggests that energy transfer from STE to Ce does not play a major role despite a good overlap between STE emission and Ce-absorption. The substantial decrease in STE excitation at wavelengths at which Ce-excitation increases (153 nr:) clearly indicates that the Ce-excitation mechanism does not involve STE states but, on the contrary, actually competes with them even reducing the formatior and contribution of STEs in the presence of cerium. All the evidence is consistent with a Ce-excitation mechanism involving mostly free holes and electrons.

In Fig. 2 we show emission and excitation spectra of $\mathrm{CaF}_{2}: 0.1 \% \mathrm{Eu}$. Under gamma excitation (Fig. 2c) the emission spectrum shows two well-kr.own bands, the $d-f$ transition of $\mathrm{Eu}^{2+}$ at $422 \mathrm{~nm}$ and the STE emission at $280 \mathrm{~nm}$. Excitation spectra of these emissions reveal their different origin; at the localized center in the case of the $\mathrm{Eu}^{2+}$ (Fig. 2a) [27], and excitonic in the case of the $280 \mathrm{~nm}$ emission (Fig. 2b). The single sharp line at $115 \mathrm{~nm}$ with a huge Stokes shift of $6.4 \mathrm{eV}$ corresponds, therefore, to creation of band excitons. Under $115 \mathrm{~nm}$ excitation the emission of $\mathrm{CaF}_{2}$ : Eu (Fig. 2d) shows a dominant STE band and, in addition, 


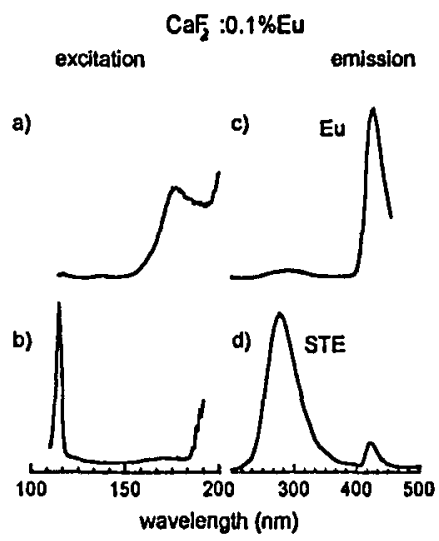

Fig. 2. The emission and excitation spectra of Eu-doped $\mathrm{CaF}_{2}$. Emission spectra: (c) the emission spectrum under gamma excitation, (d) - the emission spectrum under $115 \mathrm{~nm}$ excitation. Excitation spectra: (a) excitation spectrum of the $\mathrm{Eu}^{2+}$ emission $(422 \mathrm{~nm})$, (b) excitation spectrum of the STE emission $(290 \mathrm{~nm})$. All spectra except the gamma excited emission spectrum $(300 \mathrm{~K})$ were taken at $13 \mathrm{~K}$.

some small contribution from the $\mathrm{Eu}^{2+}$ indicating that free excitons do provide an additional channel of energy transfer. Ilowever, the gross difference between gamma and $115 \mathrm{~nm}$ excited emissions proves that the dominant mechanism of the host-to-ion energy transfer must be different.

\section{Discussion and conclusions}

While the results presented in this paper augment the notion that the radiative recombination via $\mathrm{RE}^{3+}$ ion initiated by hole binding and producing $3+$ $d-f$ emission is fairly well established in the case of $\mathrm{Ce}$, and potentially viable for some other ions, the reversed sequence process remains elusive. Obviously, when estimating the recombination energy available in such a reversed process, one has to consider an additional energy loss due to self-trapping of a hole. This loss in fluorides amounts to about 4-5 eV which, combined with the electron trap depth of about 1-2 eV, leaves 4-6 eV available for a radiative transition. The question whether this is enough to excite $d-f$ transitions of any RE-ion except $\mathrm{Ce}^{3+}$ remains open.

Since $\mathrm{Eu}^{2+}$ level in difluorides, at about $5 \mathrm{eV}$ below the conduction band [22], is positioned exceptionally low, the combined energy loss is high and no $\mathrm{Eu}^{3+}$ emission is expected [23]. Nevertheless $\mathrm{CaF}_{2}: \mathrm{Eu}$ is known to scintillate very efficiently although its emission is due to $d-f$ transition of $\mathrm{Eu}^{2+}$ even in the case of as-grown (not additively colored) material in which a $3+$ charge state prevails [28]. Based on our results we suggest the explanation assuming that $\mathrm{Eu}^{3+}$ in $\mathrm{CaF}_{2}$ captures an electron into the excited instead of the ground state of the $\mathrm{Eu}^{2+}$ which is too low for the nonradiative capture process. The filled $\mathrm{Eu}^{2+}$ level, at about $6 \mathrm{eV}$ above the valence band, is possibly too high to capture a band hole but, with the huge relaxation loss, it may be just right to bind a self-trapped hole. We conclude that 
in $\mathrm{CaF}_{2}: \mathrm{Eu}$, depending on the initial charge state of $\mathrm{Eu}$, the cycle may be initiated in two different ways both of which do eventually produce the same $d-f$ emission of the $\mathrm{Eu}^{2+}$ ion. The recombination via $\mathrm{Eu}$, although based on consecutive carrier trapping, does not provide an example of the reversed sequence process discussed in this paper.

\section{Acknowledgments}

This work has been supported by the US Department of Energy (grant No. DE-FG02-90ER61033) and the European Community (contract No. ERBCIPDCT940037). The hospitality and help of Dr. J.C. Sutherland and his team at the Brookhave National Light Source is also gratefully acknowledged. Special thanks are due to my distinguished colleagues, Prof. A. Lempicki and Prof. C. Brecher, for their continuous support, and to my young colleagues, Dariusz Wiśniewski and Jarosław Głodo, who conducted all the experiments and have always been ready to help whenever needed.

\section{References}

[1] G. Blasse, in: Heavy Scintillators for Scientific and Industrial Applications, Eds. F. De Notaristefani, P. Lecoq, M. Schneegans, Editions Frontieres, Gif-sur-Yvette 1993, p. 85.

[2] A. Lempicki, A.J. Wojtowicz, E. Berman, Nucl. Instrum. Methods Phys. Res. A 333, 304 (1993); A. Lempicki, A.J. Wojtowicz, J. Lumin. 60/61, 942 (1994).

[3] A. Lempicki, E. Berman, A.J. Wojtowicz, M. Balcerzyk, L.A. Boatner, IEEE Trans. Nucl. Sci. 40, 384 (1993).

[4] See contributions of H. Newman, T. Matulewicz, H. Hilke, A.J. Dean, B.C. Grabmaier, C.L. Melcher, M. Goldberg, in: Heavy Scintillators for Scientific and Industrial Applications, Eds. F. De Notaristefani, P. Lecoq, M. Schneegans, Editions Frontieres, Gif-sur-Yvette 1993.

[5] C.C. Klick, J.H. Schulman, Solidi State Phys. 5, 97 (1957).

[6] A.J. Wojtowicz, A. Lempicki, D. Wiśniewski, L.A. Boatner, in: Scintillator and Phospher Materials, Eds. M.J. Weber, P. Lecoq, R.C. Ruchti, C. Woody, W.M. Yen, R. Zhu, in series: MRS Symposium Proc., Vol. 348, MRS, San Francisco 1994, p. 123.

[7] A.J. Wojtowicz, A. Lempicki, S. Wiśniewski, M. Balcerzyk, C. Brecher, Conference Record, NSS BMIC, IEEE, Norfolk 1994, Ed. R. Trendler, Vol. 1, IEEE Piscateway, 1995, p. 134; A.J. Wojtowicz, A. Lempicki, D. Wiśniewski, M. Balcerzyk, C. Brecher, IEEE Trans. Nucl. Sci., in print.

[8] A.J. Wojtowicz, in: Inorganic Scintillators and their Applications, Proc. Intern. Conf. SCINT95, Eds. P. Dorenbos, C. van Eijk, Delft University Press, The Netherlands, Delft 1996, p. 94.

[9] K.S. Song, R.T. Williams, Self-Trapped Excitons, Springer Verlag, Berlin, Heidelberg 1993.

[10] M.V. Korzhik, W.P. Trower, Appl. Phys. Lett. 66, 2327 (1995).

[11] R.C. Taylor et al., IEEE Trans. Nucl. Sci. 33, 243 (1986).

[12] K.A. Kalder, F.A. Malysheva, Opt. Spectrosc. 31, 135 (1971).

[13] G. Blasse, J. Chem. Phys. 45, 2356 (1966).

[14] N.F. Mott, A.M. Stoneham, J. Phys. C, Solid State Phys. 10, 3391 (1977). 
[15] V.N. Kadchenko, M. Elango, Phys. Status Solidi A 46, 315 (1978); T.A. Green, M.E. Riley, P.M. Richards, G.M. Loubriel, D.R. Jennison, R.T. Williams, Phys. $B$ 39, 5407 (1989).

[16] M.J. Norget, A.M. Stoneham, J. Phys. C, Solid State Phys. 6, 238 (1973).

[17] R.G. Kaufman, W.B. Hadley, H.N. Hersh, IEEE Trans. Nucl. Sci. 17, 82 (1970).

[18] M.S. Abdrakhamanov, A.E. Aluker, B.Z. Gorbenko, R.G. Deich, G.S. Dumbadze, Sov. Phys. Sol. St. 31, 895 (1989).

[19] Y. Suzuki, M. Ilirai, Semicond. Insul. 5, 445 (1983).

[20] D.J. Robbins, P.J. Dean, Adv. Phys. 27, 499 (1978).

[21] G.K. Wertheim, A. Rosenzweig, R.L. Cohen, H.J. Guggenheim, Phys. Rev. Lett. 27, 505 (1971).

[22] C. Pedrini, F. Rogemont, D.S. McClure, J. Appl. Phys. 59, 1196 (1986).

[23] J.L. Merz, P.S. Pershan, Phys. Rev. 162, 217 (1967); ibid 162, 235 (1967).

[24] D. Wiśniewski, W. Drozdowski, A.J. Wojtowicz, A. Lempicki, P. Dorenbos, J.T.M. de Haas, C.W.E. van Eijk, A.J.I. Bos, presented at II Intern. Winter Workshop on Spectroscopy and Structure of Rare Earth Systems, Polanica 1996, Proc. of this Conf. (Part II), Acta Phys. Pol. A 90, (1996).

[25] A. Lempicki, M.H. Rondles, D. Wiśniewski, M. Balcerzyk, C. Brecher, A.J. Wojtowicz, IEEE Trans. Nucl. Sci. 42, 280 (1995).

[26] R.L. Wood, W. Hayes, J. Phys. C, Solid Stale Phys. 15, 7209 (1982).

[27] E. Loh, Phys. Rev. 175, 533 (1968).

[28] N.N. Ershov, T.I. Nikitinskaya, V.M. Reiterov, P.A. Rodnyi, L.M. Trofimova, S.I. Yurkov, Opt. Spectrosc. 45, 935 (1978). 